\title{
COISOMETRIC EXTENSIONS OF CONTRACTION SEMIGROUPS
}

\author{
WACLAW SZYMANSKI
}

\begin{abstract}
One more approach to dilation theorems for contraction semigroups is presented. The result is obtained by applying the *-dilation theorem for *-semigroups to a *-semigroup with the algebraic operation defined in a special way.
\end{abstract}

1. Introduction. The purpose of this note is to present still another approach to the dilation problem for contraction semigroups in Hilbert spaces. Its general idea can be viewed as a counterpart of Sz.-Nagy's method of the proof of the Halmos-Bram characterization of subnormal operators [3]. The convenient and reasonably general setting for this approach seems to be the one following Mlak [1], where special subsemigroups of ordered groups are treated. Most of the known theorems concerning contraction semigroups give their unitary dilations rather than coisometric extensions, although these two concepts are closely related. Therefore, as far as the existence of a coisometric extension of a contraction semigroup is concerned, the result of this paper is known. But the method to obtain it is quite different from the known ones. The classical Sz.-Nagy approach to this problem for one contraction carried over later by Mlak to the case of ordered groups involves the application of the dilation theorem for groups to a special positive definite function constructed from a contraction. As a consequence a unitary dilation of a contraction (contraction semigroup) is obtained [4, Chapter I. 8, 1, Theorem 2.1]. In what follows a slightly unusual *-semigroup will be constructed and the *-dilation theorem will be applied. This leads directly to a coisometric extension of a semigroup of contractions.

2. Preliminaries. $L(H)$ stands for the algebra of all linear, bounded operators in a complex Hilbert space $H$. The identity map in $H$ will be denoted by $I_{H}$. A semigroup $S$ is called a *-semigroup if an involution (i.e. a mapping ${ }^{*}: S \rightarrow S$ such that $\left.(s t)^{*}=t^{*} s^{*},\left(s^{*}\right)^{*}=s, s, t \in S\right)$ is defined on $S$. It will be assumed that *-semigroups have unit 1 satisfying $1^{*}=1$. Let $S$ be a ${ }^{*}$-semigroup. A function $\varphi: S \rightarrow L(H)$ is called positive definite (PD) if

$$
\sum\left(\varphi\left(t^{*} s\right) f(s), f(t)\right) \geq 0
$$

for all functions $f$ in the family $F(S, H)$ of functions from $S$ to $H$ vanishing off a finite subset of $S$. A function $\varphi: S \rightarrow L(H)$ is called *-dilatable if there is a Hilbert space $K$, an involutions preserving semigroup homomorphism $\pi: S \rightarrow L(K)$ satisfying $\pi(1)=I_{K}$ and a linear bounded mapping $R: H \rightarrow K$ such that

$$
\varphi(s)=R^{*} \pi(s) R ; \quad s \in S .
$$

Received by the editors April 3, 1985 and, in revised form, May 30, 1985.

1980 Mathematics Subject Classification. Primary 47A20, 47D05.

Key words and phrases. Contraction semigroup, coisometry, *-dilation. 
Such a triple $(K, \pi, R)$ is called a *-dilation of $\varphi$.

*Dilation Theorem [3, Principal TheOREM, 2]. Let $S$ be a ${ }^{*}$-semigroup. A function $\varphi: S \rightarrow L(H)$ is ${ }^{*}$-dilatable if and only if $\varphi$ is $P D$ and there is a function $c: S \rightarrow \mathbf{R}$ and a constant $\alpha \geq 0$ such that

(a) $c(s t) \leq c(s) c(t), s, t \in S$,

(b) $\|\varphi(s)\| \leq \alpha c(s), s \in S$.

An operator $V \in L(H)$ is called a coisometry if $V V^{*}=I_{H}$. If $S$ is a semigroup, a semigroup homomorphism $\rho: S \rightarrow L(H)$ will be called coisometric if each $\rho(s)$ is a coisometry, $s \in S$. In particular, if $V \in L(H)$ is a coisometry and $\mathbf{N}$ denotes the additive semigroup of all nonnegative integers, then the mappping $\rho: \mathrm{N} \rightarrow$ $L(H), \rho(n)=V^{n}, n \in \mathbf{N}$ is a coisometric semigroup homomorphism. Let $G$ be a commutative group with the algebraic operation denoted by + and the unit denoted by 0 . Suppose that $G_{+}$is a subsemigroup of $G$ that satisfies: $G_{+} \cup\left(-G_{+}\right)=G$, $G_{+} \cap\left(-G_{+}\right)=\{0\}$. Then $G_{+}$induces a total order relation in $G$ defined by the condition: $m \leq n$ if $n-m \in G_{+}, n, m \in G$. In such a case it will be said that $G$ is ordered by the subsemigroup $G_{+}$. Conversely, every total order relation $\leq$ in $G$ determines uniquely the subsemigroup $G_{+}$of all nonnegative elements which satisfies the above conditions. $G=\mathbf{Z}$ and $G=\mathbf{R}$ are two typical and most natural examples of such a group.

3. The result. Let $G$ be a commutative group ordered by the subsemigroup $G_{+}$. In the set $G_{+} \times G_{+}$define:

an involution: $(m, n)^{*}=(n, m), m, n \in G_{+}$,

an algebraic operation: for $(j, k),(m, n) \in G_{+} \times G_{+}$:

$$
(j, k) \#(m, n)= \begin{cases}(m+j-n, k) & \text { if } j \geq n, \\ (m, k+n-j) & \text { if } j \leq n .\end{cases}
$$

Proposition 1. The structure $\left(G_{+} \times G_{+}, \#,{ }^{*}\right)$ is a ${ }^{*}$-semigroup with the unit $(0,0)$. The mapping $G_{+} \rightarrow G_{+} \times G_{+}$defined by $n \rightarrow(n, 0), n \in G_{+}$is a 1-1 semigroup homomorphism.

ProOF. The main point is to prove that the operation \# is associative. Here are the results of calculations: If $(j, k),(m, n),(p, q) \in G_{+} \times G_{+}$, then

$$
\begin{aligned}
& ((j, k) \#(m, n)) \#(p, q) \\
& \quad= \begin{cases}(p+m+j-n-q, k) & \text { if } j \geq n, m+j-n \geq q, \\
(p, k+q-(m+j-n)) & \text { if } j \geq n, m+j-n \leq q, \\
& \text { or } j \leq n, m \leq q, \\
(p+m-q, k+n-j) & \text { if } j \leq n, m \geq q,\end{cases}
\end{aligned}
$$

and

$$
\begin{aligned}
(j, k) \#((m, n) \#(p, q)) \\
\quad= \begin{cases}(p+m+j-n-q, k) & \text { if } m \geq q, j \geq n \text { or } m \leq q, j \geq n+q-m, \\
(p, k+n+q-m-j) & \text { if } m \leq q, j \leq n+q-m \\
(p+m-q, k+n-j) & \text { if } m \geq q, j \leq n\end{cases}
\end{aligned}
$$

Now the associativity follows. The remaining assertions can be checked without difficulty. Notice that $(j, k) \#(m, j)=(m, k)$ if $j, k, m \in G_{+}$. Q.E.D. 
The ${ }^{*}$-semigroup $\left(G_{+} \times G_{+}, \#,{ }^{*}\right)$ will be denoted by $G^{\#}$. The semigroup $G_{+}$ can be treated as a subsemigroup of $G^{\#}$. The next proposition describes operator valued *-semigroup homomorphisms of $G^{\#}$, i.e. the semigroup homomorphisms from $G^{\#}$ into the multiplicative semigroup $L(K)$ which preserve involutions. The proof is a direct consequence of the definition of \#.

Proposition 2. (a) If $\rho: G^{\#} \rightarrow L(K)$ is a ${ }^{*}$-semigroup homomorphism, then $\rho_{0}: G_{+} \rightarrow L(K)$ defined by $\rho_{0}(n)=\rho(n, 0), n \in G_{+}$, is a coisometric semigroup homomorphism and $\rho(m, n)=\rho_{0}(n)^{*} \rho_{0}(m),(m, n) \in G^{\#}$.

(b) If $\rho_{0}: G_{+} \rightarrow L(K)$ is a coisometric semigroup homomorphism then $\rho: G^{\#} \rightarrow$ $L(K)$ defined by $\rho(m, n)=\rho_{0}(n)^{*} \rho_{0}(m),(m, n) \in G^{\#}$ is a ${ }^{*}$-semigroup homomorphism.

This proposition makes it clear why the operation \# has been introduced.

Let $S$ be a semigroup. A semigroup homomorphism $\pi: S \rightarrow L(H)$ will be said to have a coisometric extension if there is a Hilbert space $K$ and a coisometric semigroup homomorphism $\rho_{0}: S \rightarrow L(K)$ such that $H \subset K, H$ is invariant for each $\rho_{0}(s), s \in S$, and $\pi(s)=\rho_{0}(s) \mid H$. The following remark will be used. It is stated and proved here for the sake of completeness.

REMARK [3]. Suppose $H, K$ are Hilbert spaces, $H \subset K, P$ is the orthogonal projection of $K$ onto $H$ and $A \in L(K)$. The following conditions are equivalent:

(i) $H$ is invariant for $A$,

(ii) if $B=P A \mid H$, then $B^{*} B=P A^{*} A \mid H$,

(iii) if $B=P A \mid H$, then $B^{* n} B^{m}=P A^{* n} A^{m} \mid H$ for all $m, n \in \mathbf{N}$.

ProOF. Once (ii) $\Rightarrow$ (i) is proved, everything follows. Let $x \in H$. Since $B=$ $P A \mid H,\|B x\|=\|P A x\|$. Since $B^{*} B=P A^{*} A \mid H,\|B x\|^{2}=\|A x\|^{2}$. Q.E.D.

Now everything is prepared to prove the following

THEOREM. Suppose $G$ is a commutative group ordered by a subsemigroup $G_{+}$. Let $\pi: G_{+} \rightarrow L(H)$ be a semigroup homomorphism. Define $\varphi: G^{\#} \rightarrow L(H)$ by $\varphi(m, n)=\pi(n)^{*} \pi(m),(m, n) \in G^{\#}$. Then the following conditions are equivalent:

(i) $\|\pi(n)\| \leq 1$ for each $n \in G_{+}$,

(ii) $\varphi$ is $P D$ on $G^{\#}$,

(iii) $\varphi$ is ${ }^{*}$-dilatable,

(iv) $\pi$ has a coisometric extension.

Moreover, (iii) and (iv) are related as follows: If $(K, \rho, R)$ is $a^{*}$-dilation of $\varphi$, then $H \subset K, R: H \rightarrow K$ is the isometric embedding and $\rho_{0}: G_{+} \rightarrow L(K)$ defined by $\rho_{0}(n)=\rho(n, 0), n \in G_{+}$, is a coisometric extension of $\pi$. Conversely, if $\rho_{0}: G_{+} \rightarrow L(K)$ is a coisometric extension of $\pi$, if $R: H \rightarrow K$ is the inclusion map and if $\rho: G^{\#} \rightarrow L(K)$ is defined by $\rho(m, n)=\rho_{0}(n)^{*} \rho_{0}(m),(m, n) \in G^{\#}$, then $(K, \rho, R)$ is $a^{*}$-dilation of $\varphi$.

PROOF. (iv) $\Rightarrow(\mathrm{i})$ is clear.

(iii) $\Rightarrow$ (iv). Let $(K, \rho, R)$ be a *-dilation of $\varphi . \rho: G^{\#} \rightarrow L(K)$ is a *-semigroup homomorphism. By Proposition 2(a), $\rho_{0}: G_{+} \rightarrow L(K)$ defined by $\rho_{0}(n)=\rho(n, 0)$, $n \in G_{+}$, is a coisometric semigroup homomorphism and $\rho(m, n)=\rho_{0}(n)^{*} \rho(m)$, $(m, n) \in G^{\#} .(K, \rho, R)$ is a *-dilation of $\varphi$, hence

$$
\pi(n)^{*} \pi(m)=\varphi(m, n)=R^{*} \rho(m, n) R=R^{*} \rho_{0}(n)^{*} \rho_{0}(m) R, \quad m, n \in G_{+} .
$$


Since $R^{*} R=\varphi(0,0)=I_{H}, H$ can be treated as a subspace of $K$ and $P=R^{*}$ as the orthogonal projection of $K$ onto $H$. Thus,

$$
\pi(n)^{*} \pi(m)=P \rho_{0}(n)^{*} \pi_{0}(m) \mid H, \quad m, n \in G_{+} .
$$

For each arbitrarily fixed $m \in G_{+}$this equality gives $\pi(m)=P \rho_{0}(m) \mid H(n=0)$ and $\pi(m)^{*} \pi(m)=P \rho_{0}(m)^{*} \rho_{0}(m) \mid H(n=m)$.

By the above remark, $H$ is invariant for $\rho_{0}(m)$ and, clearly, $\pi(m)=\rho_{0}(m) \mid H$. Thus $\pi$ has a coisometric extension $\rho_{0}$.

(i) $\Rightarrow$ (ii). Take $\beta=(m, n), \gamma=(k, j) \in G^{\#}$. Then

$$
\begin{aligned}
& \varphi\left(\gamma^{*} \# \beta\right)=\varphi((j, k) \#(m, n))= \begin{cases}\varphi(m+j-n, k) & \text { if } j \geq n, \\
\varphi(m, k+n-j) & \text { if } j<n,\end{cases} \\
& = \begin{cases}\pi(k)^{*} \pi(m) \pi(j-n) & \text { if } j \geq n, \\
\pi(n-j)^{*} \pi(k)^{*} \pi(m) & \text { if } j<n .\end{cases}
\end{aligned}
$$

If $f \in F\left(G^{\#}, H\right)$, then

$$
\begin{aligned}
\Omega= & \sum_{\beta, \gamma}\left(\varphi\left(\gamma^{*} \# \beta\right) f(\beta), f(\gamma)\right) \\
= & \sum_{\substack{m, k \\
j \geq n}}(\pi(m) \pi(j-n) f(m, n), \pi(k) f(k, j)) \\
& +\sum_{\substack{m, k \\
j<n}}(\pi(m) f(m, n), \pi(n-j) \pi(k) f(k, j)) .
\end{aligned}
$$

Define $h: G_{+} \rightarrow H$ by $h(n)=\sum_{p} \pi(p) f(p, n), n \in G_{+}$. Then $h \in F\left(G_{+}, H\right)$ and

$$
\Omega=\sum_{j \geq n}(\pi(j-n) h(n), h(j))+\sum_{j<n}(h(n), \pi(n-j) h(j)) .
$$

It is proved in [1, Theorem 2.1] in the general case (and in [4, Chapter I. 8] in case $G=\mathbf{Z}$ ) that if (i) holds, then $\Omega \geq 0$ for each $h \in F\left(G_{+}, H\right)$. To get exactly the same form of $\Omega$ as it stands in [1], $\pi(n)^{*}$ has to be taken instead of $\pi(n), n \in G_{+}$. Thus $\varphi$ is PD on $G^{\#}$.

(ii) $\Rightarrow$ (i). Fix $n_{0} \in G_{+}$and define $T=\pi\left(n_{0}\right)$. Then $T^{\nu}=\pi\left(\nu n_{0}\right)$ for each $\nu \in \mathbf{N}$. Take $g \in F(\mathbf{N}, H)$ and consider

$$
\Omega^{\prime}=\sum_{\nu \geq \mu}\left(T^{\nu-\mu} g(\mu), g(\nu)\right)+\sum_{\nu<\mu}\left(g(\mu), T^{\mu-\nu} g(\nu)\right) .
$$

If $\varphi$ is PD on $G^{\#}$, then $\Omega^{\prime} \geq 0$ for each $g \in F(\mathbf{N}, H)$. It follows from [4, Chapter I. 8] that $\left\|T^{*}\right\|=\|T\| \leq 1$.

(i) $\Rightarrow$ (iii). The constant function $c: G^{\#} \rightarrow \mathbf{R}, c(m, n)=1,(m, n) \in G^{\#}$ and the constant $\alpha=1$ satisfy conditions (a), (b) of the ${ }^{*}$ Dilation Theorem. Thus $\varphi$ is *-dilatable on $G^{\#}$. (It has been observed in [2, p. 31] that every PD, norm-bounded function on a *-semigroup is *-dilatable.) The last statement of the Theorem follows from Proposition 2(b). Q.E.D.

Finally, a contraction semigroup will be considered on a subsemigroup of an ordered group, which is isomorphic neither to $\mathbf{Z}$ nor to $\mathbf{R}$. 
EXAMPLE. Fix a real, irrational number $\alpha$. The set $G=\{\alpha m+n: m, n \in \mathbf{Z}\}$ with the addition is a subgroup of the group $\mathbf{R}$. The group $G$ is ordered by the subsemigroup $G_{+}=\{\alpha m+n \in G: \alpha m+n \geq 0\}$. The mapping $(m, n) \rightarrow \alpha m+n$, $m, n \in \mathbf{Z}$ establishes a group isomorphism of the group $\mathbf{Z}^{2}=\mathbf{Z} \times \mathbf{Z}$ with the usual addition onto $G$. Thus $\mathbf{Z}^{2}$ can be ordered by the subsemigroup $\mathbf{Z}_{+}^{2}=\{(m, n) \in$ $\left.\mathbf{Z}^{2}: \alpha m+n \geq 0\right\}$. The above group isomorphism becomes an order-isomorphism. Now fix a selfadjoint operator $S \in L(H)$, a nonzero positive operator $P \in L(H)$ and two real numbers $a, b$. Define $\pi: G_{+} \rightarrow L(H)$ by

$$
\pi(\alpha m+n)=\exp (-(\alpha m+n) P+i(a m+b n) S), \quad \alpha m+n \in G_{+} .
$$

It is immediate that $\pi$ is a semigroup homomorphism of $G_{+}$into the multiplicative semigroup $L(H)$. Each $\pi(\alpha m+n), \alpha m+n \in G_{+}$, is a contraction, because $\|\exp (B)\| \leq 1$ if and only if $\operatorname{Re} B \leq 0$ for an arbitrary operator $B \in L(H)$. Now the Theorem implies that the corresponding function $\varphi$ defined in its statement is *-dilatable on the *-semigroup $G^{\#}$ and that $\pi$ has a coisometric extension.

ACKNOWLEDGMENTS. I would like to thank Professor John B. Conway for inviting me to Indiana University and for his great hospitality. I also thank him and Professor Ciprian Foias for encouraging discussions concerning this paper.

\section{REFERENCES}

1. W. Mlak, Unitary dilations in case of ordered groups, Ann. Polon. Math. 17 (1966), 321328.

2. F. H. Szafraniec, Dilations on involution semigroups, Proc. Amer. Math. Soc. 66 (1977), 30-32.

3. B. Sz.-Nagy, Extensions of linear transformations in Hilbert spaces which extend beyond the space, Appendix to F. Riesz and B. Sz.-Nagy, Functional Analysis, Ungar, New York, 1960.

4. B. Sz.-Nagy and C. Foias, Harmonic analysis of operators in Hilbert spaces, NorthHolland, Amsterdam, London; Akad. Kiadó, Budapest, 1970.

Department of Applied Mathematics, Academy of Agriculture, Krakow, POLAND 47405

DEPARTMENT OF MATHEMATICS, INDIANA UNIVERSity, BloOMINGTON, INDIANA

Current address: Department of Mathematical Sciences, West Chester University, West Chester, Pennsylvania 19383 